\title{
Charmonium Decays at BESIII
}

\author{
Houbing Jiang ${ }^{a, *}$ \\ ${ }^{a}$ Shandong University, \\ Jimo Binhailu 72, Qingdao 266237, P. R. China \\ E-mail: jianghb@mail.sdu.edu.cn
}

The relative simplicity of the lowest-lying charmonium states can be used as benchmarks to test the QCD and QCD-inspired models, as these states lay in the transition region between perturbative and non-perturbative QCD. While the properties and many decay channels of some charmonium states below the open-charm threshold (such as $h_{c}$ or $\eta_{c}(2 S)$ ) are still far from being known, many high-mass charmonium states above the open charm threshold predicted by potential models are not been found yet. Since 2009, BESIII has been scanning and investigating the charmonium spectrum to shed light on the internal structure of charmonium states. Thanks to its unique $J / \psi$, $\psi(2 S)$ and even large XYZ data sets, BESIII could overcome statistical limitations. Recent results on charmonium decays from BESIII are presented.

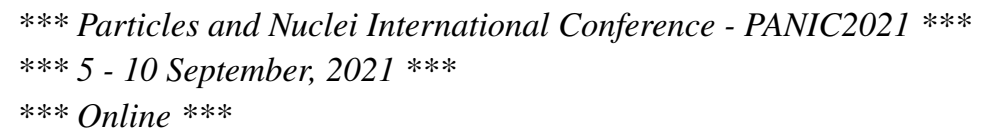

${ }^{*}$ Speaker 


\section{Introduction}

The relative simplicity of the low-mass charmonium states allows precision tests of various theoretical techniques, ranging from models to approximations of QCD to numerical calculations of the full QCD Lagrangian (LQCD). Whilst charmonium states are just located in the transition region between perturbative QCD and nonperturbative QCD, it provides a unique and important perspective on the dynamics of strong force physics [1]. However, the spectrum of charmonium states with masses above the open charm threshold is apparently more complicated. There are still many excited states that have not found yet. And the past decades have seen the discovery of a large number of new states that are still yet to be satisfactorily understood. Search and study of the high-mass states is also crucial to the understanding of exotic particles.

The BESIII studies of charmonium states includes spectroscopy, measuring transitions between charmonium states, and searching for new decay channels. Moreover, the light charmonium states are primarily studied using large and clean samples of $\psi(3686)$ or $J / \psi$ decays, the excited charmonium states can be produced using higher-energy collisions. This article is organized as follows. First, The decays of charmonium $\rightarrow B \bar{B}+X$ are shown, where $X$ could be any particle. Second, we present the results of the decays about charmonium $\rightarrow M+X$. Then, the recent results about transitions between charmonium states are shown. Finally, the summary is presented.

\section{Charmonium $\rightarrow B \bar{B}+X$}

\subsection{The study of $\psi(3686) \rightarrow \bar{\Sigma}^{0} \Lambda+c . c$.and $\chi_{c J(J=0,1,2)} \rightarrow \Lambda \bar{\Lambda}$}

Charmonium decays to baryon pairs provide a novel method to explore the properties of baryons [2]. In this work, a sample of $4.481 \times 10^{8} \psi(3686)$ events is used to measure the branching fraction of $\psi(3686) \rightarrow \bar{\Sigma}^{0} \Lambda+c . c$., with $\bar{\Sigma}^{0} \rightarrow \gamma \bar{\Lambda}, \bar{\Lambda}(\Lambda) \rightarrow \bar{p} \pi^{+}\left(p \pi^{-}\right)$[3]. The $\Lambda \bar{\Lambda}$ pair decay from the P-wave charmonium $\chi_{c J}$ states are also stuied with the same final states. The branching fraction of $\mathcal{B}\left(\psi(3686) \rightarrow \bar{\Sigma}^{0} \Lambda+\right.$ c.c. $)=(1.60 \pm 0.31 \pm 0.13 \pm 0.58) \times 10^{-6}$, the third uncertainty is the one due to interference with the continuum. Compared with the result using CLEO-c data [4], $(12.3 \pm 2.4) \times 10^{-6}$, this result is siginificantly smaller but consistent with the theoretical prediction [5] within $1 \sigma$. The branching fractions of $\chi_{c J} \rightarrow \Lambda \bar{\Lambda}$ are listed in Table 1, the third uncertainties are the one due to the uncertainties on the $\psi(3686) \rightarrow \gamma_{\chi_{c} J}$ branching fractions. These results are consistent with the world average values, but not with the theoretical predictions [6].

\subsection{Measurements of the branching fractions of $\chi_{c J(J=0,1,2)} \rightarrow n K_{S}^{0} \Lambda+c . c$.}

The color-octet mechanism, which successfully describes several decay patterns of P-wave $\chi_{c J}$ states [7], may be applicable to further $\chi_{c J}$ decays. Meaurements of $\chi_{c J}$ hadronic decays can provide new input on the color-octet mechanism. In this work, the decays of $\chi_{c J} \rightarrow n K_{S}^{0} \Lambda+$ c.c. are observed for the first time using the $4.481 \times 10^{8} \psi(3686)$ data sample, the $\chi_{c J}$ are produced via $\psi(3686) \rightarrow \gamma \chi_{c J}$ [8]. The measured branching fractions of $\chi_{c J} \rightarrow n K_{S}^{0} \Lambda+c . c$. are summarized in Table 1 . Isospin symmetry is examined by comparing the results with the isospin conjugate decays of $\chi_{c J} \rightarrow p K^{-} \bar{\Lambda}+$ c.c. [9]. The ratios $\frac{\mathcal{B}\left(\chi_{c J} \rightarrow p K^{-} \bar{\Lambda}+c . c .\right)}{\mathcal{B}\left(\chi_{c J} \rightarrow n K_{S}^{0} \Lambda+c . c .\right)}$ are also listed in Table 1, and no obvious isospin violation is observed. 
Table 1: Summary of the branching fractions of $\chi_{c J} \rightarrow \Lambda \bar{\Lambda}, \chi_{c J(J=0,1,2)} \rightarrow n K_{S}^{0} \Lambda+$ c.c. decays and the ratios of $\frac{\mathcal{B}\left(\chi_{c J} \rightarrow p K^{-} \bar{\Lambda}+c . c .\right)}{\mathcal{B}\left(\chi_{c J} \rightarrow n K_{S}^{0} \Lambda+c . c .\right)}$.

\begin{tabular}{cccc}
\hline \hline Mode & $\mathcal{B}\left(\chi_{c J} \rightarrow \Lambda \bar{\Lambda}\right)\left(\times 10^{-4}\right)$ & $\mathcal{B}\left(\chi_{c J} \rightarrow n K_{S}^{0} \Lambda+c . c\right)\left(\times 10^{-4}\right)$ & $\frac{\mathcal{B}\left(\chi_{c J} \rightarrow p K^{-} \bar{\Lambda}+c . c .\right)}{\mathcal{B}\left(\chi_{c J} \rightarrow n K_{S}^{0} \Lambda+c . c .\right)}$ \\
\hline$\chi_{c 0}$ & $3.64 \pm 0.10 \pm 0.10 \pm 0.07$ & $6.65 \pm 0.26 \pm 0.41$ & $1.98 \pm 0.09 \pm 0.14$ \\
$\chi_{c 1}$ & $1.31 \pm 0.06 \pm 0.06 \pm 0.03$ & $1.66 \pm 0.12 \pm 0.12$ & $2.71 \pm 0.24 \pm 0.20$ \\
$\chi_{c 2}$ & $1.91 \pm 0.08 \pm 0.17 \pm 0.04$ & $3.58 \pm 0.16 \pm 0.23$ & $2.35 \pm 0.14 \pm 0.16$ \\
\hline \hline
\end{tabular}

\subsection{Measurement of branching fractions of $\psi(3686)(J / \psi) \rightarrow \Sigma^{+} \bar{\Sigma}^{-}$}

Measurements on the decays of $J / \psi$ and $\psi(3686$ ) can be used to study flavor-SU(3) symmetry breaking. In addition, tests of the $12 \%$ rule using the baryonic decay modes may be helpful in understanding the $\rho \pi$ puzzle [10]. In this work, the branching fraction of $\psi(3686)(J / \psi) \rightarrow \Sigma^{+} \bar{\Sigma}^{-}$ are measured based on $1310.6 \times 10^{6} \mathrm{~J} / \psi$ and $448.1 \times 10^{6} \psi(3686)$ events [11]. The branching fractions of $J / \psi$ and $\psi(3686)$ decaying to $\Sigma^{+} \bar{\Sigma}^{-}$are measured to be $(10.61 \pm 0.04 \pm 0.36) \times 10^{-4}$ and $(2.52 \pm 0.04 \pm 0.09) \times 10^{-4}$, both are in agreement with the previous measurement [4] within 2 standard deviations. The ratio $\mathcal{B}\left(\psi(3686) \rightarrow \Sigma^{+} \bar{\Sigma}^{-}\right) / \mathcal{B}\left(J / \psi \rightarrow \Sigma^{+} \bar{\Sigma}^{-}\right)$is calculated to be $(23.8 \pm 1.1) \%$. The result also violates the "12\% rule".

\section{Charmonium $\rightarrow M+X$}

\subsection{Measurement of the inclusive branching fraction for $\psi(3686) \rightarrow K_{S}^{0}+$ anything}

The sum of the branching fractions of $\psi(3686)$ for all the decay channels is only approximately $90 \%$, indicating that there are still many decay modes missing. Measurements of the branching fractions of inclusive $\psi(3686)$ decays can guide the search for new exclusive decay modes. In this analysis, the observed cross sections of $e^{+} e^{-} \rightarrow K_{S}^{0}+$ anything (where $\mathrm{X}=$ anything) are measured at $\sqrt{s}=3.640 \sim 3.701 \mathrm{GeV}[12]$. By fitting the observed cross sections as a function of the center-of-mass energy, the $\mathcal{B}\left(\psi(3686) \rightarrow K_{S}^{0} X\right)$ is determined to be $(16.04 \pm 0.29 \pm 0.90) \%$. The sum of all the branching fraction of $\psi(3686)$ decays to exclusive $K_{S}^{0}$ final states is $\sim 5.95 \%$, which is much lower than the current measurement. This suggests that there are many undiscovered exclusive channels for $\psi(3686)$ decay to final states containing $K_{S}^{0}$.

\subsection{Observation of $\eta_{c} \rightarrow \eta \eta \eta^{\prime}$}

In this work, the $\eta_{c}$ is searched in the $\eta \eta \eta^{\prime}$ invariant mass distribution base on $1310.6 \times 10^{6}$ $J / \psi$ events [13]. The $\eta^{\prime}$ is reconstructed via the decay channels $\eta^{\prime} \rightarrow \gamma \pi^{+} \pi-$ and $\eta^{\prime} \rightarrow \pi^{+} \pi^{-} \eta$, and $\eta$ via the decay channel $\gamma \gamma$.A clear $\eta_{c}$ signal is observed for the first time, the product branching fraction of $\mathcal{B}\left(J / \psi \rightarrow \gamma \eta_{c}, \eta_{c} \rightarrow \eta \eta \eta^{\prime}\right)$ is determined to be $(4.86 \pm 0.62 \pm 0.45) \times 10^{-5}$, which is compatible with the theoretical prediction [14]. 


\section{Charmonium $\rightarrow$ Charmonium $+X$}

\subsection{Search for new decay modes of $\psi_{2}(3823)$}

The motivation of this analysis is to provide additional experimental evidence for the correct assignment of the $\psi_{2}$ (3823) to be the $J=2$ spin-triplet partner, by comparing its decay channels to the theoretical predictions. In this work, the decays $\psi_{2}(3823) \rightarrow \gamma \chi_{c 0, c 1, c 2}, \pi^{+} \pi^{-} J / \psi, \pi^{0} \pi^{0} J / \psi$, $\eta J / \psi$, and $\pi^{0} J / \psi$ are searched for using the process $e^{+} e^{-} \rightarrow \pi^{+} \pi^{-} \psi_{2}(3823)$ in a $19 \mathrm{fb}^{-1}$ data sample at $\sqrt{s}=4.1 \sim 4.7 \mathrm{GeV}$ [15]. The process $\psi_{2}(3823) \rightarrow \gamma \chi_{c 1}$ is confirmed with the high significance of $11.8 \sigma$, and an evidence for the process $\psi_{2}(3823) \rightarrow \gamma_{\chi_{c 2}}$ is found with a significance of $3.2 \sigma$. No significant $\psi_{2}$ (3823) signals are observed for other channels. And the measured branching fraction ratios $\frac{\mathcal{B}\left(\psi_{2}(3823) \rightarrow \ldots\right)}{\mathcal{B}\left(\psi_{2}(3823) \rightarrow \gamma \chi_{c 1}\right)}$ are presented in Table 2.

Table 2: The branching fraction ratios $\frac{\psi_{2}(3823) \rightarrow \gamma \chi_{c 2}}{\psi_{2}(3823) \rightarrow \gamma \chi_{c 1}}$ for different $\psi_{2}(3823)$ decay channels.

\begin{tabular}{cc}
\hline \hline Channels & $\frac{\mathcal{B}\left(\psi_{2}(3823) \rightarrow \ldots\right)}{\mathcal{B}\left(\psi_{2}(3823) \rightarrow \gamma \chi_{c 1}\right)}$ \\
\hline$\gamma \chi_{c 2}$ & $0.28_{-0.11}^{+0.14} \pm 0.02$ \\
$\pi^{+} \pi^{-} J / \psi$ & $<0.06$ \\
$\pi^{0} \pi^{0} J / \psi$ & $<0.11$ \\
$\eta J / \psi$ & $<0.14$ \\
$\pi^{0} J / \psi$ & $<0.03$ \\
$\gamma \chi_{c 0}$ & $<0.24$ \\
\hline \hline
\end{tabular}

\subsection{Measurement of $e^{+} e^{-} \rightarrow \gamma \chi_{c J(J=0,1,2)}$ cross sections}

The radiative transitions between high-mass charmonium(-like) and $\chi_{c J(J=0,1,2)}$ can be used to search for high-mass states and also help to understand the nature of these states. In this work, the cross sections of $e^{+} e^{-} \rightarrow \gamma \chi_{c J(J=0,1,2)}$ are measured between $\sqrt{s}=3.77-4.60 \mathrm{GeV}$ base on 19.3 $\mathrm{fb}^{-1} e^{+} e^{-}$annihilation data [16]. The processes of $e^{+} e^{-} \rightarrow \gamma \chi_{c 1, c 2}$ are observed for the first time at $\sqrt{s}=4.178 \mathrm{GeV}$ with significances of $7.6 \sigma$ and $6.0 \sigma$, respectively. The cross section line shape of $e^{+} e^{-} \rightarrow \gamma \chi_{c 1}$ is described with conventional charmonium states $\psi(3686), \psi(3770), \psi(4040)$, and $\psi(4160)$. For the $e^{+} e^{-} \rightarrow \gamma \chi_{c 2}$ process, one more resonance is added to describe the line shape with a significance of $5.8 \sigma$. Its parameters are measured to be $M=4371.7 \pm 7.5 \pm 1.8 \mathrm{MeV} / c^{2}$ and $\Gamma_{t o t}=51.1 \pm 17.6 \pm 1.9 \mathrm{MeV}$. In addition, the measured cross sections for $e^{+} e^{-} \rightarrow \gamma \chi_{c 1, c 2}$ are consistent with the potential model predictions [17]. For the $e^{+} e^{-} \rightarrow \gamma_{\chi_{c 0}}$ process, no obvious signal is observed. And the upper limits on cross section at $90 \%$ C.L. are reported.

\section{Summary}

In summary, many progress, including the hadronic decays of $\eta_{c}, \chi_{c J}, \psi(3686)$ also the transitions between high-mass charmonium and low-mass charmonium states, have been achieved in the study of charmonium decays at BESIII. Up to present, BESIII already accumulated 10 Billion $J / \psi$ event and 3 Billion $\psi(3686)$ events, and $\sim 22 \mathrm{fb}^{-1} e^{+} e^{-}$annihilation data above $3.8 \mathrm{GeV}$, and we can expect more precise measurements for the charmonium states both of above and below the open charm threshold in the next years. 


\section{References}

[1] S. L. Olsen, T. Skwarnicki and D. Zieminska, Nonstandard heavy mesons and baryons: Experimental evidence, Rev. Mod. Phys. 90, no.1, 015003 (2018).

[2] D. M. Asner et al., Charm physics, Int. J. Mod. Phys. A 24S1, 499-502 (2009).

[3] M. Ablikim et al. [BESIII], Measurements of the branching fractions of $\psi(3686) \rightarrow \bar{\Sigma}^{0} \Lambda+c . c$. and $\chi_{c J(J=0,1,2)} \rightarrow \Lambda \bar{\Lambda}$, Phys. Rev. D 103, 112004 (2021).

[4] S. Dobbs et al., Hyperon Form Factors \& Diquark Correlations, Phys. Rev. D 96, 092004 (2017).

[5] K. Zhu, X. H. Mo and C. Z. Yuan, Determination of the relative phase in $\psi^{\prime}$ and $J / \psi$ decays into baryon and antibaryon, Int. J. Mod. Phys. A 30, 1550148 (2015).

[6] R. G. Ping, B. S. Zou and H. C. Chiang, P-wave charmonium decays into baryon and antibaryon pairs in quark pair creation model, Eur. Phys. J. A 23, 129-133 (2004).

[7] S. M. H. Wong, Color octet contribution in exclusive $p$ wave charmonium decay into octet and decuplet baryons, Eur. Phys. J. C 14, 643-671 (2000).

[8] M. Ablikim et al. [BESIII], Observation of the decays $\chi_{c J} \rightarrow \mathrm{nK}_{\mathrm{S}}^{0} \bar{\Lambda}+$ c.c., JHEP 11, 217 (2021).

[9] M. Ablikim et al. [BESIII], Measurements of $\psi^{\prime} \rightarrow \bar{p} K^{+} \Sigma^{0}$ and $\chi_{c j} \rightarrow \bar{p} K^{+} \Lambda$, Phys. Rev. $D$ 87, no.1, 012007 (2013).

[10] M. E. B. Franklin et al., et al. Measurement of $\psi(3097)$ and $\psi^{\prime}$ (3686) Decays Into Selected Hadronic Modes, Phys. Rev. Lett. 51, 963-966 (1983).

[11] M. Ablikim et al. [BESIII], Measurement of branching fractions of $J / \psi$ and $\psi(3686)$ decays to $\Sigma^{+}$and $\bar{\Sigma}^{-}$,

[12] M. Ablikim et al. [BESIII], Measurement of the inclusive branching fraction for $\psi(3686) \rightarrow$ KSO+anything, Phys. Lett. B 820, 136576 (2021).

[13] M. Ablikim et al. [BESIII], Search for the X(2370) and observation of $\eta_{c} \rightarrow \eta \eta \eta^{\prime}$ in $J / \psi \rightarrow \gamma \eta \eta \eta^{\prime}$, Phys. Rev. D 103, no.1, 012009 (2021).

[14] W. I. Eshraim and C. S. Fischer, Hadronic decays of the (pseudo-)scalar charmonium states $\eta_{c}$ and $\chi_{c 0}$ in the extended Linear Sigma Model, Eur. Phys. J. A 54, no.8, 139 (2018).

[15] M. Ablikim et al. [BESIII], Search for new decay modes of the $\psi_{2}(3823)$ and the process $e^{+} e^{-} \rightarrow \pi^{0} \pi^{0} \psi_{2}$ (3823), Phys. Rev. D 103, no.9, L091102 (2021).

[16] M. Ablikim et al. [BESIII], Measurement of $e^{+} e^{-} \rightarrow \gamma \chi_{c 0, c 1, c 2}$ cross sections at center-ofmass energies between 3.77 and $4.60 \mathrm{GeV}$, Phys. Rev. D 104, no.9, 092001 (2021).

[17] T. Barnes, S. Godfrey and E. S. Swanson, Higher charmonia, Phys. Rev. D 72, 054026 (2005). 